\title{
Cocaine activates platelets and increases the formation of circulating platelet containing microaggregates in humans
}

\author{
C M Heesch, C R Wilhelm, J Ristich, J Adnane, F A Bontempo, W R Wagner
}

\begin{abstract}
Objective-To determine whether there is evidence of platelet activation following in vivo cocaine administration in humans, as cocaine abuse is associated with myocardial infarction and stroke, and platelet activation leading to thrombosis is a possible mechanism.

Setting-University hospital.

Design and subjects-Following a randomised, double blind crossover design, 14 healthy volunteers were studied twice, receiving cocaine $(2 \mathrm{mg} / \mathrm{kg}$ intranasally) once and placebo once. Flow cytometric analysis of P-selectin expression (an $\alpha$ granule membrane protein found on the surface of activated platelets), quantification of the platelet specific proteins platelet factor 4 and $\beta$ thromboglobulin, and measurement of platelet containing microaggregate and platelet microparticle (fragment) formation were used to assess platelet activation. Circulating von Willebrand factor antigen ( $\mathrm{vWF}$ ) was measured to evaluate a possible role of endothelial stimulation concurrent with platelet activation.

Results-There was an increase in both platelet factor 4 (mean (SD), 16 (7) to 39 (22) IU/ml, $\mathrm{p}=0.04)$ and $\beta$ thromboglobulin $(70(20)$ to $98(26) \mathrm{IU} / \mathrm{ml}, \mathrm{p}<0.01)$ at 120 minutes following cocaine administration. Platelet containing microaggregate formation was increased at 40 minutes (from $47(3.2) \%$ to $54(2.0) \%, \mathrm{p}<0.001)$ and 80 minutes $(55(2.5) \%, \mathrm{p}=0.04)$. Bleeding time decreased following cocaine from 10 (1) to 9 (1) minutes $(\mathrm{p}=0.07)$. No changes in any of the measured variables were noted following placebo administration.

Conclusions-Cocaine exposure causes platelet activation, $\alpha$ granule release, and platelet containing microaggregate formation. These data support the view that cocaine, even at the relatively low doses commonly self administered by occasional abusers, may promote thrombosis and predispose healthy individuals to ischaemic events. Platelet inhibitors should be considered early in any patient with suspected cocaine related ischaemia.

(Heart 2000;83:688-695)
\end{abstract}

Keywords: platelets; cocaine; flow cytometry; myocardial infarction

Department of Medicine, University of Pittsburgh Medical Center, 328 Scaife Hall, 3550 Terrace Street, Pittsburgh, PA

15261, USA

C $M$ Heesch

F A Bontempo

Department of

Surgery, University of

Pittsburgh Medical

Center

C R Wilhelm

J Ristich

W R Wagner

Department of

Molecular Genetics

and Biochemistry,

University of

Pittsburgh Medical

Center

J Adnane

Correspondence to:

Dr Wagner

email:

wagnerwr@msx.upmc.edu

Accepted 8 February 2000
The continuous increase in availability and popularity of cocaine has caused a dramatic rise in cardiac emergencies. ${ }^{1}$ Cases of myocardial infarction have received particular attention, as they often occur-as does cocaine associated sudden cardiac death - in an age group in which cardiac problems are uncommon. At necropsy, cocaine abusers often have premature and atypical coronary atherosclerotic lesions. ${ }^{2}$ Moreover, acute platelet-rich thrombi have been described in fatal cocaine related infarcts in both normal and atherosclerotic coronary vessels. ${ }^{1}$ In view of these peculiar features of cocaine induced ischaemic events, it has been proposed that this drug could activate platelets, leading to increased aggregation and thrombus formation. ${ }^{2}$ It has also been suggested that, in the setting of chronic cocaine abuse, the repeated release of cell growth factors by cocaine activated platelets might promote atherosclerosis, thus predisposing this population to thrombosis and ischaemia, even in the absence of acute intoxication. ${ }^{3}$ As the temporal association between increased platelet activation and myocardial infarction is well established, ${ }^{4}$ platelet activation by cocaine, directly or indirectly leading to thrombosis, is an intriguing hypothesis that could explain many of the cardiovascular complications seen in the setting of acute and chronic abuse of this drug.

Unfortunately, available clinical and experimental data on the interaction of cocaine with platelets and the haemostatic system are conflicting and inconclusive. Clinical data suggest that cocaine not only predisposes to thrombosis, but may also lead to haemorrhagic complications under certain conditions..$^{5-8}$ Studies directly incubating human platelets with cocaine in vitro have reported an increase, ${ }^{910}$ no change, ${ }^{11}$ and a decrease ${ }^{12}$ in aggregation, whereas animal studies using rabbit ${ }^{13}$ and dog platelets ${ }^{14}$ showed increased activation. These discrepancies in the experimental data are not surprising. Platelet responses to drugs vary significantly between species, so that the results of animal studies may not necessarily apply to humans. Studies of platelets exposed to cocaine in vitro do not reproduce the clinical scenario, as platelet activation and aggregation are complex processes, involving the interaction with other blood cells, the complement and coagulation systems, and the vascular endothelium..$^{15}$ Finally, the interpretation of data from human cocaine users is complicated by the fact that many such subjects have been chronically exposed to other 
toxins (such as alcohol or opioids), drug adulterants, or contaminated drug paraphernalia.

The question whether cocaine can indeed promote thrombosis by activating platelets is important, as this would not only confirm a postulated mechanism of cocaine related myocardial ischaemia, but also provide support for the use of platelet inhibitors such as aspirin in this setting, a treatment until now not supported by any experimental or observational data ${ }^{17}$ Accordingly, our purpose in this study was to evaluate the acute effects of cocaine on platelet function in healthy, drug naive subjects by administering cocaine in vivo and measuring in vivo platelet activation and platelet containing microaggregate formation, using flow cytometry, measurements of platelet specific proteins, and bleeding time determination.

\section{Methods}

STUDY DESIGN

After approval of the study by the biomedical institutional review board of the University of Pittsburgh Medical Center, 14 healthy volunteers ( 10 men, four women), age 23 to 41 years, gave informed consent and were enrolled in the study. All subjects denied any history of drug abuse, and all were non-smokers. None of the volunteers had taken any therapeutic drugs or other agents for at least two weeks before each study day. Negative urine pregnancy tests were obtained from all women the day before each study. Following a randomised, double blind crossover design, each subject was studied twice, receiving cocaine $2 \mathrm{mg} / \mathrm{kg}$ (Roxane Laboratories, Columbus, Ohio, USA) once, and a similar volume of saline placebo once, in random order by intranasal administration. For each individual, there was a time interval of at least two weeks between the study days.

After a restful night, the volunteers presented to the laboratory at $6 \mathrm{am}$ in the fasting state. Continuous ECG and haemodynamic monitoring was initiated. For safety purposes, intravenous access was obtained, and patency was maintained by flushing with saline. The subjects were kept in a semirecumbent position for the duration of each study.

A baseline bleeding time measurement was done on the right forearm, parallel to the long axis of the arm, using a Simplate device (Organon Teknica, Durham, North Carolina, USA). Thirty minutes later, a baseline blood sample was obtained for the determination of platelet activity. The study drug was then given. Blood sampling was repeated 40, 80, and 120 minutes later. At the end of the study, a second bleeding time determination was done.

SAMPLING PROCEDURE

Blood samples for the determination of platelet activity were obtained from peripheral veins, using slight proximal cuff pressure. For each blood withdrawal, the vein was cannulated using a 20 gauge needle connected to $20 \mathrm{~cm}$ of plastic tubing. Blood was then sampled, using gravity alone, without the application of negative pressure. The first $2 \mathrm{ml}$ of each sample were discarded, after which blood was collected into $3 \mathrm{ml}$ Monovette tubes containing 3\% sodium citrate (Sarstedt, Nümbrecht, Germany) for flow cytometric analysis and measurement of von Willebrand factor antigen (vWF). Vacutainer tubes, $3 \mathrm{ml}$ (BectonDickinson Immunocytometry Systems, San Jose, California, USA), containing an anticoagulant/antiplatelet mixture of citric acid, theophylline, adenosine, and dipyridamole (American Bioproducts, Parsippany, New Jersey, USA), were used for the measurement of platelet factor 4 and $\beta$ thromboglobulin.

FLOW CYTOMETRY

Immediately after blood collection, whole blood aliquots were incubated with saturating concentrations of purified murine monoclonal antibodies specific for the platelet marker GPIb (CD42b from Gentrak, Plymouth Meeting, Pennsylvania, USA), for the activation dependent protein P-selectin (CD62, BectonDickinson) or IgG isotype controls. CD $42 b$ is present on both quiescent and activated platelets, whereas CD62 is integral to the $\alpha$ granule membrane and expressed only upon platelet activation and subsequent secretion of granule contents. Antibodies obtained were conjugated either to fluorescein isothiocyanate (FITC) for $\mathrm{CD} 42 \mathrm{~b}$ and its IgG isotype control, or phycoerythrin (PE) for CD62 and its IgG isotype control, to allow for two colour analysis. Following incubation in the dark for 30 minutes, samples were fixed with $1 \%$ paraformaldehyde and acquired using a FACScan flow cytometer (Becton-Dickinson). For each sample, 10000 events were acquired by live gating on events positive for CD42b-FITC, with a positive fluorescence threshold determined by excluding $98 \%$ of IgG-FITC isotype control fluorescence.

On the basis of the technique of Abrams and colleagues ${ }^{18}$ single platelets and CD42b positive aggregate sized events were differentiated by using the following technique: after identification through positive fluorescence for the anti-CD 42b monoclonal antibody, the single platelet population was characterised as distinct and separate from the larger platelet containing microaggregates, based upon their degree of forward scatter; separating the populations, gates were systematically drawn around the single platelet population and the microaggregates, with R2 designated as the single platelet population and R3 as the microaggregate population, as seen in fig 1A. Platelet containing microaggregates were expressed as a percentage of the total CD $42 \mathrm{~b}$ positive population.

In a similar fashion, platelet fragments (microparticles) were identified by positive fluorescence for CD42b-FITC and forward scatter below a size threshold of $0.5 \mu \mathrm{m}$. Fluorescent calibration microspheres were used to delineate a threshold forming the boundary between $\mathrm{R} 1$, the platelet fragment population, and gate $\mathrm{R} 2$, the single platelet population.

Platelet surface expression of P-selectin was analysed by selecting the single platelet population, setting a positive fluorescence threshold to exclude $98 \%$ of IgG-PE isotype fluorescence, and measuring CD62-PE fluorescence above 

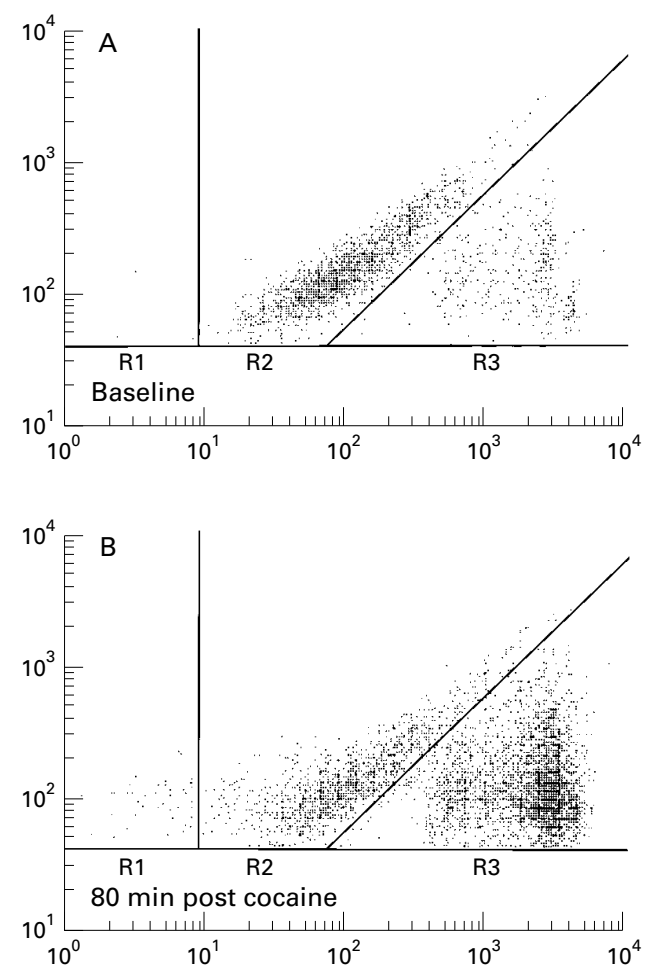

Figure 1 Representative flow cytometric tracing at $(A)$ baseline and (B) 80 minutes after cocaine administration (bottom panel). The $y$ axis measures fluorescence for the platelet marker $C D 42 b$, and the $x$ axis measures forward light scatter, both in relative units. The field to the left (R1) represents the platelet fragment population. The middle field (R2) represents the population of single platelets, and platelet containing microaggregates are contained in field $R 3$. There was a pronounced increase in microaggregates following cocaine administration.

this threshold. Activation was expressed as the percentage of the single platelet population also positive for CD62-PE, according to the methods described by Shattil and colleagues. ${ }^{19}$

PLATELET FACTOR $4, \beta$ THROMBOGLOBULIN, AND VWF DETERMINATION

Plasma concentrations of $\beta$ thromboglobulin, platelet factor 4 , and vWF were determined using commercially available enzyme linked immunosorbent assays (American Bioproducts), following standard procedures.

COCAINE AND METABOLITE MEASUREMENTS

To determine drug concentrations, samples were collected into tubes containing sodium fluoride. Analysis of cocaine and benzoylecgonine, the major metabolite of cocaine, was performed by MetPath Labs (Pittsburgh, Pennsylvania, USA), using capillary gas chromatography with electron impact mass spectrometry (GC/MS).

\section{STATISTICAL ANALYSIS}

A Wilcoxon signed rank test was used to test whether the baseline measurement was different from each subsequent time point for both the cocaine and the control data group. A nonparametric technique was used, owing to the relatively small sample size and the dependent nature of the data. In order to determine whether concentrations of cocaine and benzoylecgonine were related to the studied
Table 1 Measurements of cocaine and benzoylecgonine concentrations ( $\mathrm{ng} / \mathrm{l}$ ) following cocaine administration

\begin{tabular}{|c|c|c|c|}
\hline & \multicolumn{3}{|l|}{ Time } \\
\hline & $40 \mathrm{~min}$ & $80 \mathrm{~min}$ & $120 \mathrm{~min}$ \\
\hline Cocaine & $97.1(6.8)$ & $99.3(7.5)$ & $86.4(8.7)$ \\
\hline Benzoylecgonine & $259.3(37.4)$ & $434.3(28.2)$ & $501.4(26.3)$ \\
\hline
\end{tabular}

Values are mean (SEM).

variables across subjects, we computed Pearson correlation coefficients, averaging over the three measures of cocaine and benzoylecgonine, with the maximum difference from baseline for each variable. Similarly, the maximum blood pressure and heart rate changes from baseline were determined for each volunteer and used to assess a possible correlation between cocaine induced changes in haemodynamic variables and platelet activation.

\section{Results}

COCAINE AND METABOLITE CONCENTRATIONS

Concentrations of cocaine and benzoylecgonine are listed in table 1 . Peak cocaine concentrations were obtained at 80 minutes, consistent with the prolonged absorption process typical of the intranasal route of administration. As expected, the highest benzoylecgonine concentrations were recorded at the end of the experiment.

HAEMODYNAMIC MEASUREMENTS

Figure 2 shows the systolic and diastolic blood pressure recordings and the heart rate measurements for both the cocaine and the placebo studies. There was a modest increase in all three variables, beginning 10 minutes after the administration of cocaine. These modest, sustained changes are consistent with the drug concentration measurements, showing a slow absorption of cocaine by the nasal mucosa.

PLATELET FACTOR 4 AND $\beta$ THROMBOGLOBULIN Figure 3 shows changes in platelet factor 4 and $\beta$ thromboglobulin following the administration of cocaine and placebo. Following cocaine, platelet factor 4 values were increased from baseline values at 120 minutes $(p=0.04)$. Likewise, $\beta$ thromboglobulin concentrations were also increased from baseline at 120

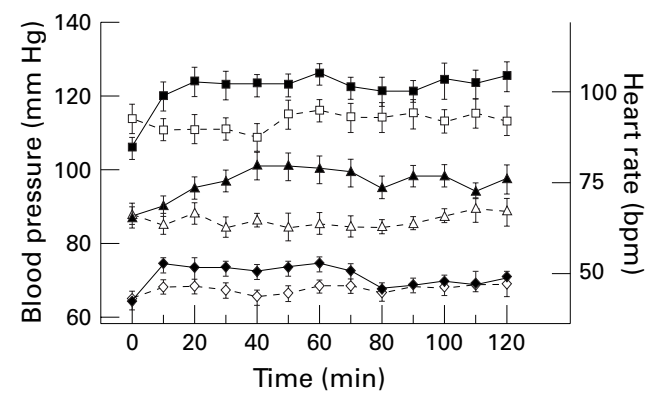

SBP cocaine $\longrightarrow$ DBP cocaine $\longrightarrow$ HR cocaine - SBP placebo - $\diamond$ - DBP placebo - - - HR placebo

Figure 2 Changes in heart rate (HR), systolic blood pressure (SPB), and diastolic blood pressure (DBP) preceding and following the administration of cocaine (black symbols) or placebo (white symbols). Values are means, error bars $=S E M$. 

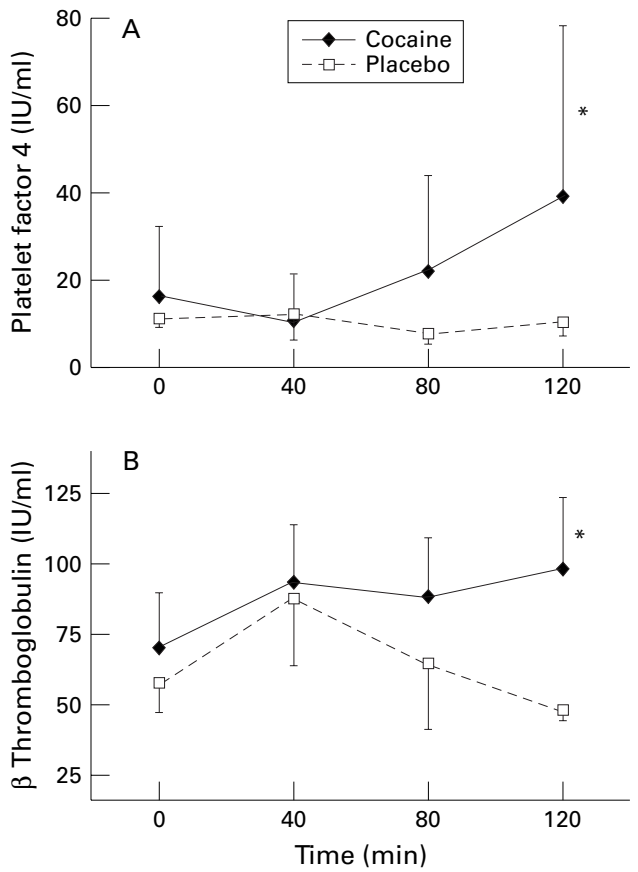

Figure 3 Changes in (A) platelet factor 4 and (B) $\beta$ thromboglobulin at 120 minutes; both variables were significantly increased from baseline. Values are means, error bars $=$ SEM.

minutes $(p=0.005)$. Following placebo, none of the subsequent measurements showed significant changes from baseline for either of these variables.

P-SELECTIN EXPRESSION AND MICROAGGREGATE FORMATION

Figure 4A shows the percentage of single platelets expressing P-selectin, following the administration of cocaine or placebo. There were no changes from baseline values with either study drug. Following cocaine, there was a significant increase in the percentage of platelet containing microaggregates at $40 \mathrm{~min}$ utes $(p=0.0005)$ and at 80 minutes $(p=0.04)$ (fig $4 \mathrm{~B}$ and demonstrated in fig 1 ). Percentages of platelet containing microaggregates were not different from baseline at any of the subsequent time points following placebo administration.

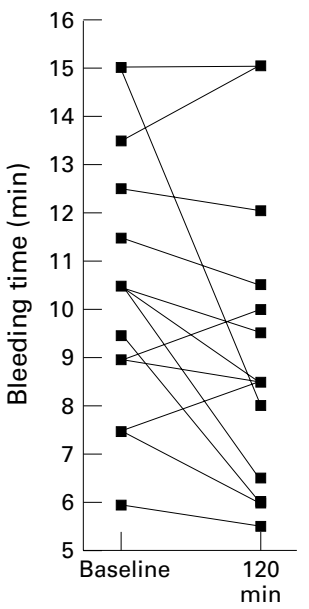

Figure 5 Results of bleeding time determinations for each individual at baseline and 120 minutes after the administration of cocaine.

\section{BLEEDING TIME MEASUREMENTS}

Figure 5 shows the bleeding time measurements before and following cocaine administration for all 14 volunteers. Bleeding time decreased in 10 volunteers, remained unchanged in one, and increased in three. Following placebo, bleeding time decreased in five, increased in eight, and remained unchanged in one subject (data not shown). Using a Wilcoxon signed rank test, bleeding time was marginally lower following cocaine $(\mathrm{p}=0.07)$; using a paired Student's $t$ test the $\mathrm{p}$ value was 0.05 . Bleeding times following placebo did not differ from baseline values.

VON WILLEBRAND FACTOR

In order to evaluate whether the activation of platelets was associated with a concomitant activation of the endothelium, we measured vWF concentrations at the four different time
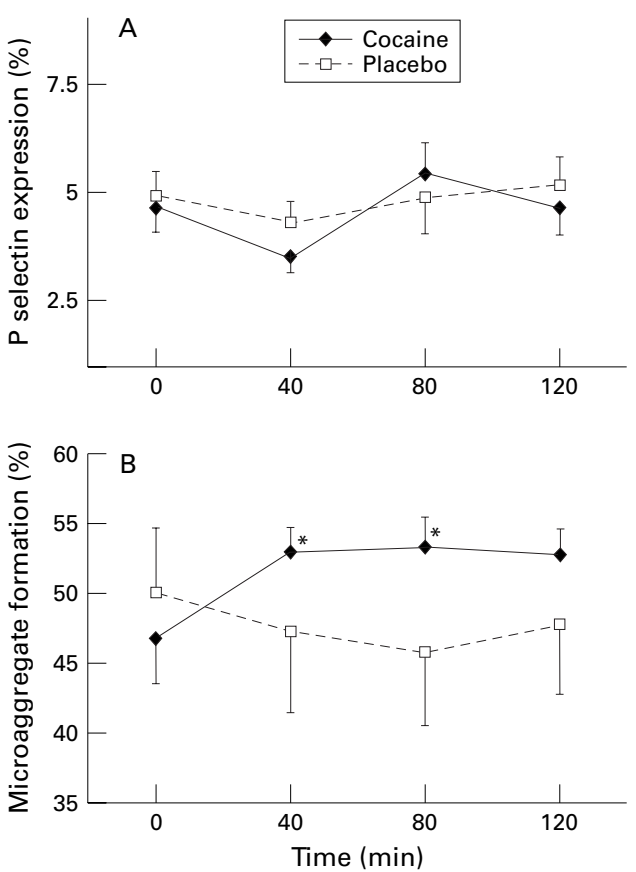

Figure 4 Changes in (A) P-selectin expression (per cent of single platelet population also positive for CD62) and (B) microaggregate formation (expressed as per cent of all $C D 42 b$ positive event). No changes were seen in P-selectin expression, whereas microaggregates were increased at 40 and 80 minutes. Values are means, error bars $=S E M$.

points. Table 2 lists the measurements of vWF following cocaine and placebo. There were no significant differences from baseline values with either study drug.

\section{PLATELET FRAGMENT FORMATION}

Platelet fragments (microparticles), defined as CD42b positive events falling below a scattering size threshold of $0.5 \mu \mathrm{m}$, are reported in table 3 before and after cocaine and placebo. There were no differences in the number of platelet fragments detected following either study drug.

\section{CORRELATION STUDIES}

Blood concentrations of cocaine and benzoylecgonine (averaged over the three measurements), as well as the maximum increases in
Table 2 Measurements of von Willebrand factor (per cent normal antigen concentration) before and after the administration of cocaine and placebo

\begin{tabular}{llll}
\hline Time & & \\
\cline { 2 - 3 } Baseline & $40 \mathrm{~min}$ & $80 \mathrm{~min}$ & $120 \mathrm{~min}$ \\
\hline
\end{tabular}

$\begin{array}{llllll}\text { Cocaine } & 118.5(7.4) & 106.0(9.1) & 110.0(9.6) & 116 & (9.9)\end{array}$ $\begin{array}{lrrrr}\text { Placebo } & 86.6(9.5) & 88.8(7.3) & 99.8(8.5) & 100.1(7.8)\end{array}$

Values are mean (SEM).

Table 3 Measurements of platelet microparticles (per cent of all $C D 42 b$ positive events) before and after the administration of cocaine and placebo

\begin{tabular}{|c|c|c|c|}
\hline Time & & & \\
\hline Baseline & $40 \mathrm{~min}$ & $80 \mathrm{~min}$ & $120 \mathrm{~min}$ \\
\hline
\end{tabular}

$\begin{array}{llllll}\text { Cocaine } & 0.59(0.17) & 0.38(0.08) & 0.40(0.08) & 0.56(0.16)\end{array}$ $\begin{array}{lllll}\text { Placebo } & 0.23(0.03) & 0.29(0.05) & 0.24(0.03) & 0.19(0.04)\end{array}$

Values are mean (SEM). 
heart rate, systolic blood pressure, and diastolic blood pressure, were evaluated with all of the above variables to assess a possible correlation between drug concentrations, haemodynamic changes, and changes in platelet activation. Changes in $\beta$ thromboglobulin correlated with an increase in heart rate $(r=0.54, \mathrm{p}=0.05)$. There was a marginal positive correlation between the average cocaine concentration and the extent of platelet containing microaggregate formation $(r=0.52, \mathrm{p}=0.08)$.

\section{Discussion}

This is the first study to show that cocaine, at doses comparable with those self administered by many "recreational" abusers, leads to platelet activation with subsequent $\alpha$ granule release, increased concentrations of circulating platelet containing microaggregates, and a slight decrease in bleeding time. The results were obtained by in vivo administration of cocaine to healthy young volunteers who did not have a history of chronic exposure to this or any other drug. The findings suggest that platelet activation may be an important mechanism contributing to ischaemic events in the setting of acute cocaine intoxication. The data also support the concept that the chronic use of cocaine could accelerate the development of atherosclerosis by stimulating the release of growth factors from platelets, as these substances - together with the platelet specific proteins $\beta$ thromboglobulin and platelet factor 4 - are contained in $\alpha$ granules and released as part of the secretory response. In addition, the chronic secretion of platelet enzymes such as elastase might contribute to vascular degeneration, because the degradation of arterial elastic tissue is a characteristic feature of atherosclerosis. ${ }^{20}$ Finally, these observations support the use of platelet inhibiting agents such as aspirin in the setting of cocaine related ischaemic syndromes.

Although we did not see an increase in the population of single platelets expressing P-selectin following cocaine, there was a significant increase in platelet specific proteins. Thus an increased turnover of $\mathrm{P}$-selectin positive cells or P-selectin shedding ${ }^{21}$ must have occurred, as this protein is expressed upon fusion of $\alpha$ granules with the cell surface during the release reaction. Furthermore, there was a significant increase in platelet containing microaggregates, which, as expected, showed positive fluorescence for CD62-PE. Thus we believe that activated single platelets may have been cleared rapidly from the circulation through platelet-platelet aggregation as well as adhesion to leucocytes and the endothelium, with subsequent removal through the reticuloendothelial system. ${ }^{22}{ }^{23}$ While we did not specifically determine the presence or absence of leucocytes in the observed $\mathrm{CD} 42 \mathrm{~b}$ positive microaggregates, unpublished data from our laboratory have shown that in subjects with activated platelets, between $10 \%$ and $50 \%$ of the microaggregate population show positivity for the leucocyte specific marker CD45.
PREVIOUS STUDIES

Early studies using hand held lenses, gross observation, and contrast microscopy suggested a decrease in aggregation when platelets were directly exposed to cocaine. ${ }^{24-27}$ Subsequent investigators ${ }^{13}$ reported both an increase and a decrease in the aggregatory response of rabbit platelets after in vitro exposure to cocaine, depending on the concentration of the drug and the choice of aggregation inducing agonist. While Rezkalla and colleagues, incubating human platelet-rich plasma samples with cocaine, found increased platelet aggregation by turbidimetry in response to $1 \mu \mathrm{M}$ ADP in a subset of samples, ${ }^{9}$ they did not observe such an increase with higher doses of ADP or any other agonist. Jennings and colleagues showed that in vitro aggregation in response to ADP, collagen, or arachidonic acid was inhibited after cocaine exposure. ${ }^{12}$ In addition, these investigators found that cocaine prevented the binding of fibrinogen to activated platelets and disrupted the organisation of the cytoskeleton. Data from our laboratory, ${ }^{28}$ on the in vitro effects of cocaine on samples from 42 human donors without a history of cocaine abuse, showed a dose dependent decrease in aggregation in response to ADP and arachidonic acid. Thus most studies directly exposing human plasma suspended platelets to cocaine have shown a decrease in the aggregatory response.

As the incubation of platelet-rich plasma samples with cocaine does not maintain the interaction of platelets with other blood cells such as leucocytes or erythrocytes, Kugelmass and colleagues evaluated platelet activation by flow cytometry of whole blood samples, to which cocaine was added. ${ }^{10}$ Using blood samples from healthy volunteers, these investigators reported a significant increase in P-selectin expression after five minutes in vitro following cocaine incubation in half of the donors.

In vivo research on the effects of cocaine on platelets has been limited. When cocaine was given intravenously to chronically instrumented conscious dogs, a substantial increase in platelet P-selectin expression was observed. ${ }^{14}$ Rinder and colleagues gave intravenous infusions of cocaine or placebo to four human volunteers with a history of cocaine abuse, who expected to receive cocaine. ${ }^{11}$ Numbers of circulating activated platelets, assessed by flow cytometry, rose significantly after infusion of either placebo or cocaine. We have studied the effects of intranasal cocaine or placebo given to healthy human volunteers without previous exposure to this drug, using ex vivo measurements of the aggregatory response of platelet-rich plasma with turbidimetry. ${ }^{29}$ There was a small but significant decrease in ex vivo platelet aggregation in response to standard agonists.

There are significant differences between these two studies of cocaine administration to humans and our present investigation. The study of Rinder and colleagues, while using similar methods (flow cytometry), differs in the population studied. The data were obtained from chronic cocaine abusers, in whom acti- 
vated platelets persisted for almost six hours after the study drug infusion. In our present study with drug naive volunteers, activated single platelets appeared to be cleared quickly from the circulation, a difference suggesting functional defects in the clearance of activated platelets following chronic cocaine abuse. Indeed, animal studies have shown that functional changes do occur in the vascular endothelium in response to chronic cocaine exposure. ${ }^{16}$ In addition, any platelet response to acute cocaine ingestion or any platelet interaction with the endothelium could have been altered by long term exposure to drugs other than cocaine.

Our own previous study of healthy volunteers, while similar in design, differed in the methods used to assess platelet function. Cocaine activates platelets in vivo, as evidenced by the production of microaggregates and the release of platelet factor 4 and $\beta$ thromboglobulin. Strongly activated platelets rapidly form microaggregates and are subsequently removed by the reticuloendothelial system. Platelets that remain in the circulation are not activated or are only partially activated and do not express surface localisation of $\mathrm{P}$-selectin. These platelets are less responsive to subsequent in vitro agonist stimulation. This is in accordance with the well known clinical observation that platelets of patients with recent thrombotic events such as myocardial infarction show decreased responsiveness. ${ }^{30}$ Avoiding these in vitro methods, the present study was able to detect the preceding in vivo platelet activation, secretion, and the formation of platelet containing microaggregates.

HAEMORRHAGIC VERSUS THROMBOTIC EFFECTS

Under certain conditions cocaine use may lead to haemorrhagic complications, which appear to occur mainly at sites of direct exposure-for example, gastrointestinal bleeding following drug ingestion, ${ }^{5}$ haemoptysis following crack inhalation, ${ }^{67}$ and mucosal bleeding following the nasal application of cocaine. ${ }^{8}$ These clinical observations support the results of most in vitro studies, showing decreased aggregation of isolated platelets following direct cocaine exposure. Jennings and colleagues postulated that cocaine may inhibit platelet function, particularly when high local concentrations are achieved. ${ }^{12}$ Thus studies directly exposing isolated platelets to cocaine in vitro, ${ }^{12} 28$ or challenging isolated platelets in vitro after previous in vivo drug exposure, ${ }^{29}$ are best suited to explain such local haemorrhagic events.

In contrast, cocaine associated thrombosis appears to result from the systemic effects of the drug and does not show a clear relation to route or timing of ingestion. In fact, myocardial ischaemic events often occur hours after cocaine abuse. The present investigationallowing for the prolonged interaction of cocaine with platelets, other blood cells, the complement system, and the vascular endothelium - was designed to model these indirect and delayed thrombotic properties of the drug.
POSSIBLE MECHANISMS

The exact mechanisms leading to platelet activation after cocaine exposure remain unknown. In this study, we evaluated the hypothesis that cocaine might stimulate platelets indirectly by activating the vascular endothelium. Of the different substances proposed as markers of endothelial activation, vWF is widely accepted. This protein, which is known to mediate platelet adhesion, is released from endothelial cells upon endothelial stimulation and in chronic vascular disease states. ${ }^{31-33}$ Although it is also a constituent of the $\alpha$ granule, platelet associated $\mathrm{vWF}$ remains attached to the surface of the platelets after the process of platelet secretion, so that circulating plasma vWF is largely a product of the vascular endothelium. ${ }^{31}$ There was no evidence of an increase in vWF following cocaine treatment, and no difference in the temporal response of vWF between the cocaine and the placebo data groups. This argues against a significant role of the vascular endothelium in the process of platelet activation by cocaine. One previous study ${ }^{34}$ reported a cocaine related increase in another substance associated with endothelial cell activation and dysfunction-plasminogen activator inhibitor-1 (PAI-1), which promotes thrombosis by inactivating tissue plasminogen activator. That study, however, enrolled patients with suspected coronary disease. Moreover, PAI-1 is less accepted as a specific marker of endothelial cell function, because it is also produced by hepatocytes, platelets, mesothelial cells, monocytes, and smooth muscle cells. ${ }^{33}$ Thus, while there may be a role for the endothelium in the process of cocaine induced platelet activation in other populations - such as patients with vascular disease, or long term cocaine abusers with possible endothelial dysfunction-our data do not provide evidence of significant endothelial stimulation by short term cocaine exposure in healthy, drug naive subjects.

Given the fact that the direct exposure of platelets to cocaine in vivo inhibits aggregation, and given the time delay in the detection of activated platelets after cocaine administration to animals, other investigators ${ }^{14}$ have suggested that it is not cocaine but its metabolites that induce platelet activation in vivo. While we did not see any effects of the major cocaine metabolites benzoylecgonine and ecgonine methyl ester at low doses on platelet aggregation in vitro, ${ }^{28}$ we cannot exclude an effect of these metabolites in the clinical setting. Moreover, we cannot rule out the accumulation of some of the drug in the liver ${ }^{35}$ with subsequent breakdown to metabolites other than those produced by serum cholinesterases, ${ }^{36}$ especially as the intranasal administration of cocaine involves some oral drug ingestion in almost all individuals, a route of administration which favours hepatic metabolism. Given the timing of the observed changes in the variables studied, however, we believe that this is less likely.

A cocaine induced alteration in endothelial antithrombotic activity has been proposed, as cocaine has been shown to increase the production of prostaglandins, with a dispropor- 
tionate increase in thromboxane relative to prostacyclin. ${ }^{16}$ While such a disturbance in the endothelial prostacyclin-thromboxane balance, with resultant enhancement of platelet aggregation, ${ }^{37}$ would be more likely to apply to chronic cocaine abusers than to our naive subjects, some in vitro evidence ${ }^{13}$ suggests that even short term exposure might alter the relation of thromboxane and prostaglandin production by platelets and vascular tissue.

One possible explanation for our observation of platelet activation could be cocaine induced haemodynamic changes with a resultant increase in shear stress on platelets. Although cocaine did transiently alter haemodynamics, animal studies ${ }^{14}$ suggest that modest haemodynamic changes such as seen in our study are not sufficient to result in platelet activation. Second, we did not find an increase in circulating vWF, a molecule released by the endothelium in conditions of increased shear stress. Third, there is evidence that shear stress increases the percentage of microparticles through platelet fragmentation. ${ }^{38}$ We did not see any cocaine associated changes in the percentage of platelet fragments, as detected by size distribution measurements. Thus we believe that in our study haemodynamic changes and shear stress are less likely to have contributed to platelet activation. Lastly, Rinder and colleagues inferred a conditioned cue sympathomimetic response as an explanation for the increase in platelet activation following both placebo and cocaine in their study of cocaine addicts, all of whom expected to receive cocaine. ${ }^{11}$ These investigators did not observe any platelet activation in healthy volunteers receiving intravenous dextrose, and in chronic cocaine abusers knowingly receiving dextrose. Thus it is conceivable that in chronic abusers the anticipation of cocaine, but not necessarily the drug itself, is associated with platelet activation, whereas in naive subjects the drug, and not the mere possibility of receiving it, causes this effect. The mechanism of such a possible conditioned platelet response remains to be determined.

\section{STUDY LIMITATIONS}

In this study we enrolled cocaine naive, healthy volunteers. This is both a strength and a weakness of the study. The findings may not necessarily apply to chronic drug abusers. On the other hand, the effects of any study drug in habitual drug abusers may be altered by comorbid conditions and the concomitant or previous use of unknown quantities of other street drugs and drug adulterants. Moreover, catastrophic cocaine induced complications are well described in occasional and first time users of the drug. For these reasons, we felt that the chosen population was best suited to test our hypothesis.

While the study followed a double blind design, some of the subjects experienced central nervous system (CNS) effects after cocaine administration which revealed that the active drug had been given. The investigators supervising the drug administration also noticed these CNS effects, but the individuals processing the blood samples were blinded to this knowledge. The only way to have overcome the potential unblinding of subjects owing to the CNS effects of cocaine would have been to test the drug against a placebo substance proven to have similar CNS effects and no platelet effects. As no such substance is known, we decided to follow the example set by previous in vivo cocaine investigations, ${ }^{34}{ }^{39-42}$ testing intranasal cocaine against intranasal saline.

\section{CONCLUSIONS}

In this study we showed that cocaine, even at relatively low doses comparable with those commonly self administered by occasional abusers, leads to an increase in platelet activation, platelet $\alpha$ granule release, and the formation of platelet containing microaggregates in healthy, drug naive subjects. The data suggest that platelet activation may be an important mechanism contributing to ischaemic complications following cocaine exposure, and add to mounting evidence disproving the widely held belief that occasional cocaine abuse poses little risk. The risk of thrombosis, similar to the risk of sudden cardiac death, is real and may affect even the first time user of small quantities of the drug. Finally, these findings provide support for the early use of platelet inhibiting agents such as aspirin, a treatment previously of unproven value in suspected cocaine related ischaemic syndromes.

1 Kolodgie FD, Farb A, Virmany R. Pathobiological determinants of cocaine-associated cardiovascular syndromes. Hum Pathol 1995;26:583-6.

2 Kloner RA, Hale S, Alker K, et al. The effects of acute and chronic cocaine use on the heart. Circulation 1992;85:40719.

3 Kolodgie FD, Virmani R, Cornhill, et al. Increase in atherosclerosis and adventitial mast cells in cocaine abusers: an alternative mechanism of cocaine-associated coronary vasospasm and thrombosis. F Am Coll Cardiol 1991;17: 1553-60.

4 Tofler GH, Brezinski D, Schafer AI, et al. Concurrent morning increase in platelet aggregability and the risk of myocardial infarction and sudden cardiac death. $\mathrm{N} \mathrm{Engl} \mathcal{F}$ Med 1987:316:1514 an

5 Riggs D, Weibly RE. Acute hemorrhagic diarrhea and cardiovascular collapse in a young child owing to environmentally vascular collapse in a young child owing to environment
acquired cocaine. Pediatr Emerg Care 1991;7:154-5.

6 Murray RJ, Albin RJ, Mergner W, et al. Diffuse alveolar hemorrhage temporally related to cocaine smoking. Chest 1988;93:427-9

7 Bailey ME, Fraire AE, Greenberg SD, et al. Pulmonary histopathology in cocaine abusers. Hum Pathol 1994;25: 203-7.

8 Anderton JM, Nassar WY. Topical cocaine and general anaesthesia: an investigation of the efficacy and side effects
of cocaine on the nasal mucosae. Anaesthesia 1975;30:80917.

9 Rezkalla SH, Mazza JJ, Kloner RA, et al. Effects of cocaine on human platelets in healthy subjects. Am f Cardiol 1993; 72:243-6.

10 Kugelmass AD, Oda A, Monahan $\mathrm{K}$, et al. Activation of human platelets by cocaine. Circulation 1993;88:876-83.

1 Rinder HM, Ault KA, Jatlow PI, et al, Platelet $\alpha$-granule release in cocaine users. Circulation 1994;90:1162-7.

12 Jennings LK, White MM, Sauer CM, et al. Cocaine-induced Jennings LK, White MM, Sauer CM, et al.
platelet defects. Stroke 1993;24:1352-9.

13 Togna G, Tempesta E, Togna AR, et al. Platelet responsiveness and biosynthesis of thromboxane and prostacyclin in response to in vitro cocaine treatment. Haemostasis 1985;15:100-7

14 Kugelmass AD, Shannon RP, Yeo EL, et al. Intravenous cocaine induces platelet activation in the conscious dog. Circulation 1995;91:1336-40.

15 Kugelmass AD, Ware JA. Cocaine and coronary artery thrombosis [letter]. Ann Intern Med 1992;116:776-7.

16 Eichhorn EJ, Demian SE, Alvarez LG, et al. Cocaineinduced alterations in prostaglandin production in rabbit aorta. $\mathcal{F}$ Am Coll Cardiol 1992;19:696-703.

17 Hollander JE. The management of cocaine-associated myocardial ischemia. N Engl f Med 1995;333:1267-72.

18 Abrams CS, Ellison N, Budzynski AZ, et al. Direct detection Abrams CS, Ellison N, Budzynski AZ, et al. Direct detection
of activated platelets and platelet-derived microparticles in of activated platelets and platelet-d
humans. Blood 1990;75:128-38.

19 Shattil SJ, Cunningham M, Hoxie JA. Detection of activated platelets in whole blood using activation-dependent monoclonal antibodies and flow cytometry. Blood 1987;70:307-15. 
20 Niewiarowski S, Holt JC, Cook JJ. Biochemistry and physiology of secreted platelet proteins. In: Colman RW, Hirsh J, Marder VJ, et al, eds. Hemostasis and thrombosis: basic principles and clinical practice. Philadelphia: JB Lippincott, 1994:546-56.

21 Michelson AD, Barnard MR, Hechtman HB, et al. In vivo tracking of platelets: circulating degranulated platelets rapidly lose surface p-selectin but continue to circulate and function. Proc Natl Acad Sci USA 1996;93:11877-82.

22 Rinder HM, Bonan JL, Rinder CS, et al. Dynamics of leukocyte-platelet adhesion in whole blood. Blood 1991;78: 1730-7.

23 Rinder HM, Bonan JL, Rinder CS, et al. Activated and unactivated platelet adhesion to monocytes and neutrophils. Blood 1991;78:1760-9.

24 O'Brien, JR. The adhesiveness of platelets and its prevention. $\mathcal{F}$ Clin Pathol 1961;14:140-9.

25 O'Brien JR. Platelet aggregation. F Clin Pathol 1962;15:446-55.

26 Aledort LM, Niemetz J. Dissociation of platelet aggregation from clot retraction, potassium loss, and adenosine triphosphate activity. Proc Soc Exp Biol Med 1968;128:658-60.

27 Geerdink P, Levy-Toledano S, Wessels H, et al. Influence of lithium on aggregation, release-reaction and function of lithium on aggregation, release-reaction and function

28 Heesch CM, Steiner M, Hernandez JA, et al. Effects of cocaine on human platelet aggregation in vitro. $\mathcal{F}$ Toxicol Clin Toxicol 1996;34:673-84.

29 Heesch CM, Negus BH, Steiner M, et al. Effects of in vivo cocaine administration on human platelet aggregation. $\mathrm{Am}$ f Cardiol 1996;78:237-9.

30 O'Brien JR, Etherington MD, Shuttleworth RD, et al. Platelet function in acute myocardial infarction patients compared with controls. Thromb Haemost 1980;44:96-9.

31 Blann AD. Is raised von Willebrand factor a marker of endothelial cell damage? Med Hypotheses 1993;41:419-24.
32 Blann AD, McCollum CN. Von Willebrand factor, endothelial cell damage and atherosclerosis. Eur ₹ Vasc Surg 1994;8:10-15. 33 Blann AD, Taberner DA. A reliable marker of endothelial cell dysfunction: does it exist? Br F Haematol 1995;90:244-8.

34 Moliterno DJ, Lange RA, Gerard RD, et al. Influence of intranasal cocaine on plasma constituents associated with endogenous thrombosis and thrombolysis. $\mathrm{Am} 7 \mathrm{Med}$ 1994;96:492-6.

35 Wiggins RC, Rolsten C, Ruiz B, et al. Pharmacokinetics of cocaine: basic studies of route, dosage, pregnancy and lactation. Neurotoxicology 1989;10:367-82.

36 Boelsterli UA, Göldlin C. Biomechanisms of cocaineinduced hepatocyte injury mediated by the formation of reactive metabolites. Arch Toxicol 1991;65:351-60.

37 Heng MCY, Haberfeld G. Thrombotic phenomena associated with intravenous cocaine. $7 \mathrm{Am}$ Acad Dermatol 1987;16:462-8.

38 Slack SM, Jennings LK, Turitto VT. Platelet size distribution measurements as indicators of shear stress-induced platelet aggregation. Ann Biomed Eng 1994;22:653-9.

39 Boehrer JD, Moliterno DJ, Willard JE, et al. Hemodynamic effects of intranasal cocaine in humans. $7 \mathrm{Am}$ Coll Cardiol 1992;120:90-3.

40 Brogan WC, Lange RA, Glamann B, et al. Recurrent coronary vasoconstriction caused by intranasal cocaine: possible role for metabolites. Ann Intern Med 1992;116:556-61.

41 Brogan WC, Lange RA, Kim AS, et al. Alleviation of cocaine-induced coronary vasoconstriction by nitroglycerin. F Am Coll Cardiol 1991;18:581-6.

42 Lange RA, Cigarroa RG, Yancy CW, et al. Cocaine-induced coronary artery vasoconstriction. $N$ Engl f Med 1989;321: $1557-62$

\section{IMAGES IN CARDIOLOGY}

\section{Echocardiographic features of cardiac involvement in Fabry's disease}

A 57 year old man was admitted to hospital in Osaka, Japan because of exertional dyspnoea and progressive renal failure. He had a history of renal dysfunction for 39 years without treatment. The patient's cousin had been diagnosed with Fabry's disease, and his sister with severe left ventricular hypertrophy with obstructive outflow tract treated with a permanent pacemaker. The $\alpha$ galactosidase A activity of his leucocytes was much less than the normal range. Fabry's disease was diagnosed by means of detailed examinations. Cross sectional echocardiography in the parasternal long axis view (top) and apical four chamber view (bottom) showed that both the interventricular septum and the left ventricular free wall were severely thickened without obstructing the left ventricle, especially in the region of the septum.

In homozygous men and some heterozygous women, glycosphingolipid is deposited in the skin, kidneys, vascular system, and heart by deficiency of the lysosomal enzyme $\alpha$ galactosidase A. Characteristically, patients die during the fourth or fifth decade from cardiac, vascular, or renal involvement. The feature of cardiac involvement is increased left ventricular wall thickness resulting from progressive glycosphingolipid deposition in the myocardium. Recently, $\alpha$ galactosidase gene mutations including partial deletions and point mutations have been detected. Molecular genetic analysis, measurement of $\alpha$ galactosidase A activity, and endomyocardial biopsy are useful to distin-
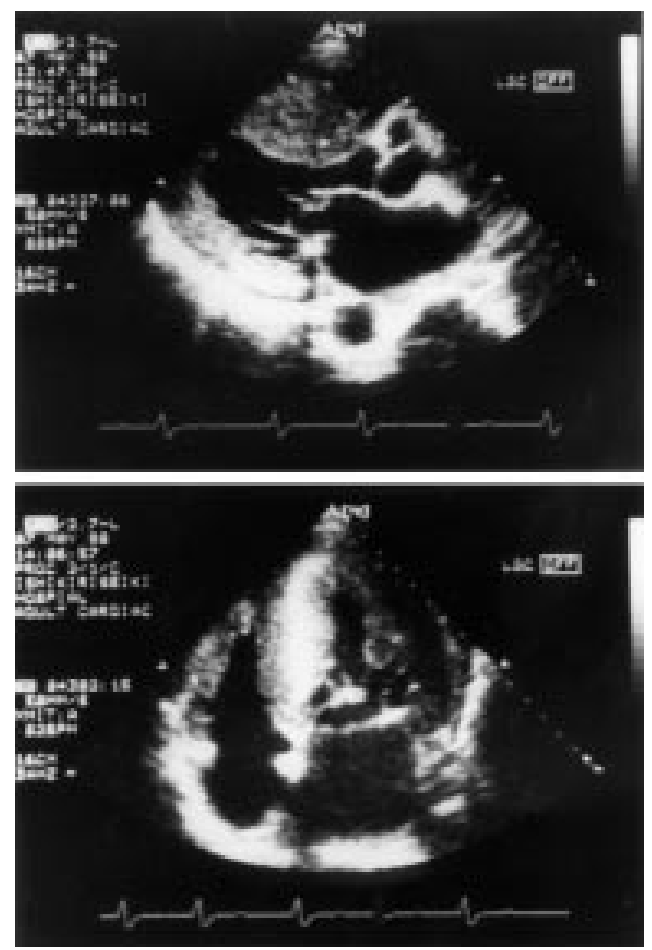

guish Fabry's disease from hypertrophic cardiomyopathy.

YASUNORI NAKAYAMA KEI TSUMURA KIYOMICHI YOSHIMARU 